\title{
Breather Wave Molecules
}

\begin{tabular}{|c|c|}
\hline Item Type & Article \\
\hline Authors & $\begin{array}{l}\text { Xu, Gang; Gelash, Andrey; Chabchoub, Amin; Zakharov, Vladimir; } \\
\text { Kibler, Bertrand }\end{array}$ \\
\hline Citation & $\begin{array}{l}\text { Xu, G., Gelash, A., Chabchoub, A., Zakharov, V., \& Kibler, B. } \\
\text { (2019). Breather wave molecules. Physical Review Letters, 122(8), } \\
084101 .\end{array}$ \\
\hline DOI & 10.1103/PhysRevLett. 122.084101 \\
\hline Publisher & AMER PHYSICAL SOC \\
\hline Journal & PHYSICAL REVIEW LETTERS \\
\hline Rights & (C) 2019 American Physical Society \\
\hline Download date & 04/01/2021 12:24:11 \\
\hline Version & Final published version \\
\hline Link to Item & http://hdl.handle.net/10150/632960 \\
\hline
\end{tabular}




\title{
Breather Wave Molecules
}

\author{
Gang Xu, ${ }^{1, \dagger}$ Andrey Gelash, ${ }^{2,3, \dagger}$ Amin Chabchoub, ${ }^{4}$ Vladimir Zakharov, ${ }^{5,6}$ and Bertrand Kibler ${ }^{1, *}$ \\ ${ }^{1}$ Laboratoire Interdisciplinaire Carnot de Bourgogne (ICB), UMR 6303 CNRS-Université Bourgogne Franche-Comté, \\ 21078 Dijon, France \\ ${ }^{2}$ Novosibirsk State University, Novosibirsk 630090, Russia \\ ${ }^{3}$ Institute of Thermophysics, SB RAS, Novosibirsk 630090, Russia \\ ${ }^{4}$ Centre for Wind, Waves and Water, School of Civil Engineering, The University of Sydney, Sydney, New South Wales 2006, Australia \\ ${ }^{5}$ Landau Institute for Theoretical Physics RAS, Chernogolovka 142432, Russia \\ ${ }^{6}$ University of Arizona, Tucson, Arizona 857201, USA
}

(Received 31 October 2018; published 27 February 2019)

\begin{abstract}
We present both a theoretical description and experimental observation of the nonlinear mutual interactions between a pair of copropagative breathers in the framework of the focusing one-dimensional nonlinear Schrödinger equation. As a general case, we show that the resulting bound state of breathers exhibits moleculelike behavior with quasiperiodic oscillatory dynamics (i.e., internal coherent interactions and pulsations), while for commensurate conditions the molecule oscillations become exactly periodic. Our theoretical model is confirmed by an experimental observation of shaped moleculelike breather light waves propagating in a nearly conservative optical fiber system. Our work sheds new light on the existence of localized wave structures and recurrence dynamics beyond the multisoliton complexes.
\end{abstract}

DOI: $10.1103 /$ PhysRevLett.122.084101

Introduction.-During the last decades, the formation of localized wave structures has attracted significant research interest in a variety of conservative and dissipative systems of nonlinear science [1], including photonics, plasma physics, Bose-Einstein condensates, fluid mechanics, biology, and chemistry. For instance, the notion of a soliton [2-3], is one of the most wellknown example of studies in which the initial concept [4] has even been generalized to nonlinear dissipative systems [5]. The notion of dissipative solitons arises from the balance between dispersion or diffraction and nonlinearity (as the conventional soliton) and between gain and losses [6]. Besides their formation, their mutual interactions such as collisions and even the emergence of stable bound states have been particularly investigated in laser physics [7-8]. Such dissipative multisoliton complexes, also called photonic molecules, result from self-organization phenomena. Such localized wave structures show dynamics similar to matter molecules, such as synthesis and vibration. The build-up and transient regimes of dissipative localized structures in mode-locked fiber lasers are currently the subject of intense studies by means of real-time ultrafast measurements [9-13]. It is worth mentioning that distinct kinds of photonic molecules have been theoretically and experimentally studied by means of various models and physical systems such as saturable nonlinear bulk media [14], dispersive-managed fibers with periodically alternating dispersion [15], BoseEinstein condensates [16], time-delayed laser system [17], and quantum nonlinear media [18].
By contrast, the existence of bound states of conventional solitons (also known as multisoliton complexes [19]) in the framework of their original studies, namely integrable Hamiltonian systems like the focusing one-dimensional nonlinear Schrödinger equation (1D-NLSE) [20], have remained largely unexplored. Of course, bisolitons or higher-order soliton solutions of the NLSE, have been analyzed theoretically and numerically [19-22]. The bound states of solitons in other integrable systems such as sine-Gordon, modified Korteweg-de Vries and Gardner equations were also found [23-25]. The key features of multi-soliton complexes are the equal group-velocity of elementary solitons and the molecule-like behavior of their internal coherent interactions with exact periodic beatings. In the simplest case, the bound state of two NLS solitons is characterized only by one recurrence frequency, and constitutes a periodic solution [20]. Such structures are asymptotically unstable, since a small perturbation can result in a change of the soliton velocities. However, besides envelope solitons, the focusing 1D-NLSE admits a wider range of exact solutions in the form of breathers on finite background [26-31], for which the conventional soliton can be considered as a limiting case. As NLSE is viewed as the basic model for various systems including plasmas, optical fibers, oceans, and cold atoms, propagation and interactions of NLS breathers in weakly nonlinear media are of fundamental interest in recent theoretical and experimental studies due to their important role in the development of modulation instability (MI) and formation of NLS rogue waves [32-35]. 
In this work, we provide a generalization of the concept of bound states of NLS solitons towards moving breather waves. Note that particular bound states formed by NLS breathers were previously studied theoretically in Refs. [31] and [36], while more general investigations analyzing space-time dynamics, more particularly their characteristic frequencies which define breather molecules, are still pending. More specifically, we present the theoretical description of the breather molecule based on the exact two-breather solution of the NLSE. We find that the breather molecule exhibits quasiperiodic or periodic oscillatory dynamics according to the characteristic frequency of elementary breather. Note that compared to the soliton molecules in dissipative cavities, which are described by Ginzburg-Landau equation [7,37,38], here we study conservative fiber systems driven by the integrable NLSE; i.e., in our case the external binding potential is absent. Nevertheless, the precise control of the input optical wave parameters and high accuracy detection allow us to demonstrate the propagation of a breather molecule formed by copropagative NLS breathers in optical fibers.

Theoretical models.-Our theoretical description is based on the breather formalism obtained within the framework of the self-focusing 1D-NLSE [39],

$$
i \psi_{\xi}+\frac{1}{2} \psi_{\tau \tau}+|\psi|^{2} \psi=0,
$$

where subscripted variables stand for partial differentiations. Here $\psi$ is a wave envelope which is a function of $\xi$ (a scaled propagation distance or longitudinal variable) and $\tau$ (a commoving time, or transverse variables, moving with the wave-group velocity). This conventional form of the NLSE has exact breather solutions, characterizing solitons propagating on an unstable finite background and interacting with it. Within this framework, different coherent structures on finite background have been intensively studied in different nonlinear dispersive media, such as Akhmediev breathers, Kuznetsov-Ma solitons and, in particular, the Peregrine solitons. The background is usually modeled by a simple plane wave solution of the NLSE $\psi_{0}=A e^{i A^{2} \xi}$, where $A$ is the background amplitude which we set to unity. The background $\psi_{0}$ is unstable with respect to long-wave perturbations (modulation instability phenomena, see, e.g., Ref. [40]). Beyond the elementary first-order breather solutions, higher-order breathers can be found to describe MI wave evolution dynamics of more complex initial wave systems. In particular, a special subclass of breather solutions, the so-called super-regular breathers, may also describe the development of modulationally unstable localized background perturbations $[30,31,39,41]$. In our work we use as in Ref. [30] notations for parameters of each breather: $R, \alpha, \mu$, and $\theta . R \in(1, \infty]$ and $\alpha \in(-\pi / 2, \pi / 2)$ to characterize the main breather properties: amplitude, group velocity and period of oscillations while $\mu \in[-\infty, \infty]$ and $\theta \in[0,2 \pi]$ to describe breather's time position and phase. We study the breather molecule using the general two-breather solution of NLSE which is presented in explicit form in the Supplemental Material [42]. We also refer to Refs. [30-31] for more details on the mathematical derivations of this solution.

We study interactions of a couple of general-type moving NLS breathers [29] and each breather is characterized by group velocity $V_{g_{-} i}$ (here $i=1$ or 2 , denoting one of the two breathers),

$$
V_{g \_i}=-\sin \alpha_{i}\left(R_{i}^{4}+1\right) /\left[R_{i}\left(R_{i}^{2}-1\right)\right],
$$

and oscillation frequency $W_{i}$,

$$
W_{i}=2\left(k_{i} V_{g_{\_} i}+\delta_{i}\right),
$$

where $k_{i}=-\left(R_{i}+1 / R_{i}\right) \sin \left(\alpha_{i}\right) / 2$ and $\delta_{i}=\left(R_{i}^{2}-1 / R_{i}^{2}\right) \times$ $\cos \left(2 \alpha_{i}\right) / 4$ (see also Ref. [38]). The breather molecule is formed when the group velocities of two breathers coincide: $\quad V_{g_{-} 1}=V_{g_{-} 2}=V_{g_{\lrcorner} \text {mol }}$ (the subscript $g \_$mol denotes the "molecule group velocity") i.e., when

$$
\frac{\left(R_{1}^{2}+1 / R_{1}^{2}\right) \sin \alpha_{1}}{\left(R_{1}-1 / R_{1}\right)}=\frac{\left(R_{2}^{2}+1 / R_{2}^{2}\right) \sin \alpha_{2}}{\left(R_{2}-1 / R_{2}\right)},
$$

and relative temporal separation $\left(\mu_{1}-\mu_{2}\right)$ is small (i.e., when nonlinear interaction occurs). In addition, the behavior of the breather molecule is controlled by the relative phase parameter $\left(\theta_{1}-\theta_{2}\right)$. Figure 1 shows several examples fulfilling the constraint (4), leading to independent (a1)-(c1) or bound (a2)-(c2) propagation of breathers.

As can be noted from Figs. 1(a2)-1(c2), the complete nonlinear interaction (for a zero temporal separation) of two copropagating breathers results in a bound state with nontrivial oscillatory behavior (i.e., internal coherent interactions and pulsations). We found that this state of two copropagative breathers has four oscillation frequencies $W_{\text {mol }}$. Two of them are the individual frequencies of breathers $W_{1}, W_{2}$ and other two are their combinations: $W_{1}-W_{2}, W_{1}+W_{2}$ (see Supplemental Material [42]). For each frequency we denote the corresponding space period $E_{j}=2 \pi / W_{\text {mol }}$, where $j=1,2,(1-2),(1+2)$. When the temporal separation of the breathers $\left(\mu_{1}-\mu_{2}\right)$ is large (the case of a dissociated molecule), they propagate separately, oscillating with their own periods $E_{1}$ and $E_{2}$, as shown in Figs. 1(a1)-(c1). When the molecule is strongly associated, one of the molecule frequencies can dominate, but in the general case the behavior is quasiperiodic [see Fig. 1(a2)].

The breather molecule can exhibit exact periodic behavior for a certain choice of parameters, namely when the characteristic frequencies of two individual breathers satisfy the commensurate condition $W_{1} / W_{2}=m_{1} / m_{2}$, where $m_{1}$ and $m_{2}$ are integer numbers: 

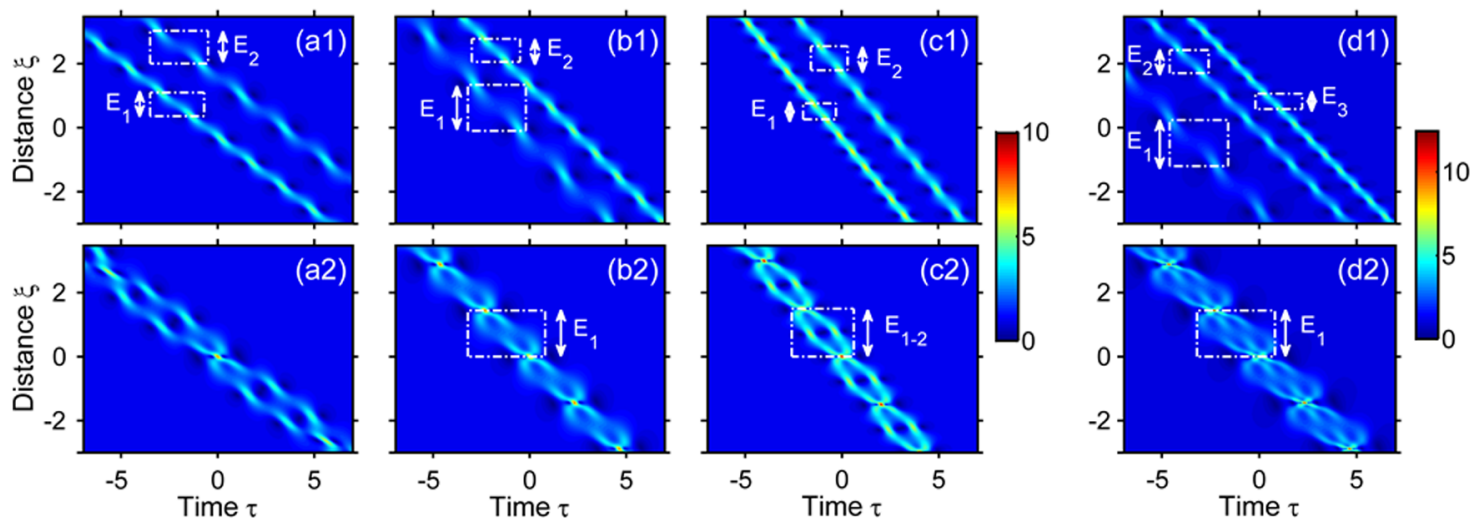

FIG. 1. False color density plots of the space-time evolution of $|\psi|$ for representative cases of (a),(b),(c) two or (d) three copropagating breathers with same group velocity, based on the $N$-breather solutions of NLSE. First column (a) $R_{1}=4, \alpha_{1}=\pi / 6, R_{2}=3.27558$, $\alpha_{2}=\pi / 5, \theta_{1,2}=0, \mu_{1}=0$, (a1) $\mu_{2}=2 \pi$, (a2) $\mu_{2}=0$. Second column (b) $R_{1}=2.75, \alpha_{1}=\pi / 6, R_{2}=4.113528, \alpha_{2}=0.376306$, $\theta_{1,2}=0, \mu_{1}=0$, (b1) $\mu_{2}=2 \pi$, (b2) $\mu_{2}=0$. Third column (c) $R_{1}=5, \alpha_{1}=\pi / 12, R_{2}=4.056606, \alpha_{2}=0.316726, \theta_{1,2}=0, \mu_{1}=0$, (c1) $\mu_{2}=2 \pi$, (c2) $\mu_{2}=0$. Fourth column (d) $R_{1}=2.75, \alpha_{1}=\pi / 6, R_{2}=4.113528, \alpha_{2}=0.376306, R_{3}=5.083628, \alpha_{3}=0.309323$, $\theta_{1,2,3}=0,(\mathrm{~d} 1) \mu_{1}=-2 \pi, \mu_{2}=0, \mu_{2}=2 \pi$, (d2) $\mu_{1,2,3}=0$.

$$
\frac{k_{1} V_{g_{-1}}+\delta_{1}}{k_{2} V_{g_{-2}}+\delta_{2}}=\frac{m_{1}}{m_{2}}
$$

Note that Eqs. (4) and (5) may have joint solutions corresponding to physically adequate breather parameters like those presented in Fig. 1 [42]. In this case, we denote $E_{\text {mol }}$ as the period of the breather molecule. As a general rule, when the ratio between $m_{1}$ and $m_{2}$ is an integer number, $E_{\mathrm{mol}}=E_{1}$ or $E_{2}$, as shown in Fig. 1(b2) with $m_{2} / m_{1}=2 / 1$. Otherwise $E_{\text {mol }}$ is equal to $E_{1-2}$ or $E_{1+2}$, see Fig. 1(c2) with $m_{2} / m_{1}=2 / 3$. For the chosen parameters $R$ and $\alpha$ of one breather, the conditions (4) and (5) lead to a high order polynomial equation that we solve numerically. Thus, to obtain such breather molecule solutions we must find parameters satisfying (4) and (5) and then use the general two-breather solution of the NLSE [42].

As mentioned, the temporal matching of the breathers $\left(\mu_{1}-\mu_{2}\right)$ is an essential parameter to generate the bound state. For a relatively large temporal separation, such as $\left(\mu_{1}-\mu_{2}\right)>\pi / 2$, the two breathers can be regarded as independent [i.e., dissociated molecule as in Figs. 1(a1)$1(\mathrm{c} 1)]$. When this value is decreased below $\pi / 2$, the mutual interaction is no longer negligible (i.e., molecule synthesis), since the characteristic frequency of the molecule breather begins to be noticeable. For a nearly zero temporal separation, a chainlike bound state is then formed between the two breathers, see Figs. 1(a2)-1(c2). This impact of the temporal matching of two co-propagating breathers is analyzed in the supplemental material [42].

In general, the breather molecule is formed by $N \geq 2$ copropagative breathers having individual frequencies $W_{j}$, where $j=1, \ldots, N$. The condition similar to Eq. (4): $V_{g_{-} 1}=V_{g_{-} 2}=\ldots=V_{g_{-} N}$ can be easily fulfilled. Such a molecule has $N^{2}$ oscillation frequencies $\left(W_{j}\right.$ and all their pairwise sums and differences) and usually demonstrates quasiperiodic behavior. One can find parameters of the $N$-breather molecule satisfying the general commensurate condition $W_{1} / W_{1} / \ldots / W_{N}=m_{1} / m_{2} / \ldots / m_{N}$ (here $m_{j}$ are integer numbers) when its oscillations are exactly periodic. An example of an exactly commensurate 3-breather molecule with the following condition $m_{3} / m_{2} / m_{1}=$ $3 / 2 / 1$ is shown in Fig. 1(d2), whereas its dissociated configuration is depicted in Fig. 1(d1). We construct this three-breather molecule using a general $N$-breather solution formula (see Refs. [30-31]) and find that the characteristic space period of this three-breather molecule is here equal to $E_{1}$.

Experiments.-Next, we performed experiments based on the propagation of arbitrarily shaped light waves in optical fibers to validate the existence of breather molecules. Our experimental setup based on high-speed telecommunication-grade components is depicted in Fig. 2. The ideal excitation of two-breather solutions of NLSE is obtained through Fourier-transform optical pulse shaping with optimized phase and amplitude controls of the optical field. Such an optical processing is based on spectral lineby-line shaping of a frequency comb source whose bandwidth can be designed specifically for the NLS solution under study. Our homemade frequency comb is generated by the implementation of a high-repetition-rate pulse source centered at $1548 \mathrm{~nm}$. This results from the 2.2$\mathrm{km}$-long nonlinear compression of a $20-\mathrm{GHz}$ modulated wave. The spectral bandwidth of the comb is finely tuned by optimizing both initial modulation depth and average power of the modulated wave before compression. Note that a phase modulator is also introduced to prevent the detrimental effect of stimulated Brillouin backscattering. Next the spectral comb passes through a programmable optical filter (wave shaper) which provides an extremely precise control of the amplitude and phase characteristics 


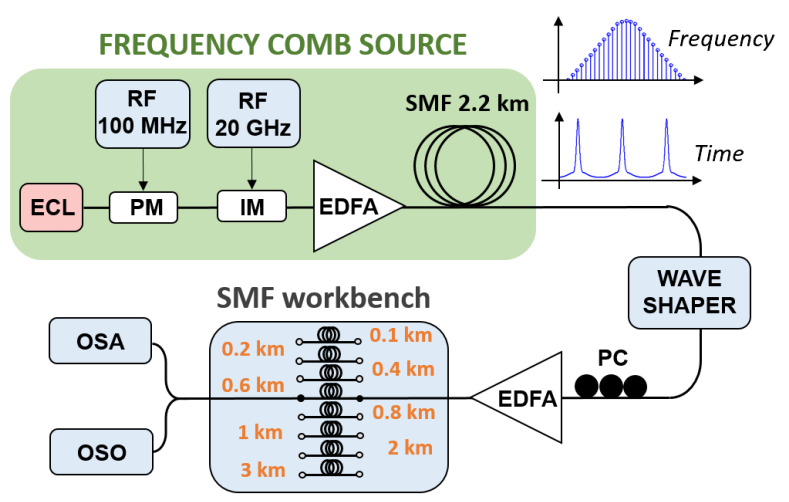

FIG. 2. Experimental setup. External cavity laser diode (ECL), phase modulator (PM), intensity modulator (Im), erbium-doped fiber amplifier (EDFA), single-mode fiber (SMF); polarization controller (PC), optical spectrum analyzer (OSA), optical sampling oscilloscope (OSO).

of each comb line. This allows us to synthesize tailored perturbations of the background wave in a time-periodic pattern whose frequency is equal to the comb spacing. Exact excitation of the two-breather solutions can be obtained in a standard single-mode fiber SMF-28 by an appropriate choice of the input average power (here $P_{0}=0.71 \mathrm{~W}$ ). Our fiber properties are the following: group velocity dispersion $\beta_{2}=-21.1 \mathrm{ps}^{2} \mathrm{~km}^{-1}$, linear losses $\alpha=0.2 \mathrm{~dB} \mathrm{~km}^{-1}$, and nonlinear coefficient $\gamma=1.2 \mathrm{~W}^{-1} \mathrm{~km}^{-1}$. The propagation of the moleculelike breather is then studied with different lengths of the same fiber and characterized by means of an optical sampling oscilloscope with subpicosecond resolution and a highdynamics-range optical spectrum analyzer. The maximum propagation distance was fixed to $3 \mathrm{~km}$, since the accumulated linear loss begins to perturb the breather dynamics for longer optical fibers. One can retrieve the correspondence between normalized and physical units by making use of the following relations between dimensional distance $z(m)$ and time $t(s)$ and previous normalized parameters: $z=\xi L_{\mathrm{NL}}$ and $t=\tau t_{0}$, where the characteristic (nonlinear) length and time scales are $L_{\mathrm{NL}}=\left(\gamma P_{0}\right)^{-1}$ and $t_{0}=$ $\sqrt{\left|\beta_{2}\right| L_{\mathrm{NL}}}$, respectively. The dimensional optical field $A(z, t)\left(W^{1 / 2}\right)$ is $A=\sqrt{P_{0}} \psi$.

In the following, we present our experimental results on the space-time evolution of a pair of copropagating breathers. We arbitrarily chose breather parameters $m_{2} / m_{1}=$ $1.98 / 1$ to be close to the commensurate condition (5). Here our goal is twofold: (i) to reveal the possible domination of specific $E_{\mathrm{mol}}$ related to exact periodic behavior, and (ii) to show typical asymmetric signatures in the breather molecule profile linked to general quasiperiodic oscillatory dynamics. Figures 3(a)-3(b) show the theoretical evolution of the breather molecule. For the zero temporal separation of two copropagating breathers, we observe a chainlike bound state that can propagate though infinite distance in
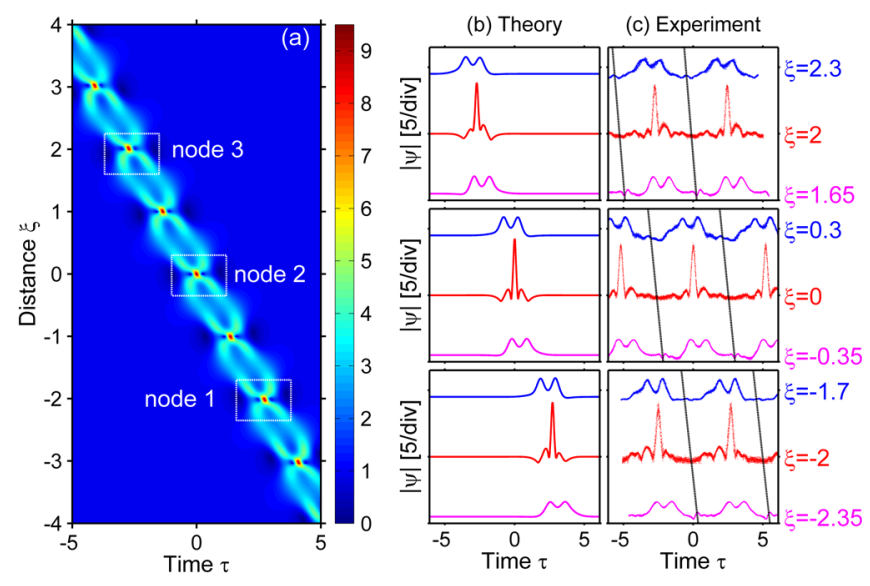

FIG. 3. Space-time evolution of a bound state of breathers $\left(R_{1}=5, \quad \alpha_{1}=0.0833 \pi, \quad R_{2}=3.5, \quad \alpha_{2}=0.1145 \pi, \quad \theta_{1,2}=0\right.$, $\mu_{1}=\mu_{2}=0$ ). (a) Two-breather solution. White rectangles denote three nodes of the breather molecule. (b) Corresponding amplitude profiles $|\psi|$. (c) Experimental measurements (square-root of recorded intensity) performed in the region of those three nodes. The periodic pattern results from our frequency-comb-based system; each element of the pattern is delimited by dashed-black lines.

the NLSE framework. However, we also notice that snapshots of the amplitude profiles, corresponding to nodes of the chain away from the origin $\xi=0$, reveal slight asymmetries [this can be also observed in the typical $X$-shape signature of the nodes in the plane $(\xi, \tau)]$. Such asymmetric amplitude profiles simply result from the general case of quasiperiodic oscillatory dynamics. We then recorded the evolution of the breather molecule around the most representative nodes, namely, for $\xi=-2,0$, and 2 . More specifically, we started nonlinear wave propagation at $\xi_{I N}=-2.35,-0.35$, and 1.65 , and we followed wave evolution by means of the different fiber lengths up to $3 \mathrm{~km}$ to reach $\xi_{\text {OUT }}=-1.7,0.3$, and 2.3 , respectively. Our wave measurements shown in Fig. 3(c) quantitatively confirm theoretical predictions of the breather molecule dynamics. The slight discrepancies between the two-breather solution and the experimental observation are ascribed to the linear fiber losses.

In order to assess the full dynamics of the breather molecule in our experiments, we provide the full evolution of both spectral and intensity profiles over one quasiperiod by combining different optical fiber segments of the SMF workbench in Fig. 2. This technique avoids the destructive cutback method required for direct measurement of the longitudinal evolution. With this method, we construct the 2D diagrams shown in Fig. 4 to depict the detailed spectral and temporal evolutions of node 2 from Fig. 3. It is worth mentioning that the maximum spectral broadening is located at the center of the node, where the maximal temporal localization of the breather pair is achieved. Again an excellent agreement is obtained with the theory about 


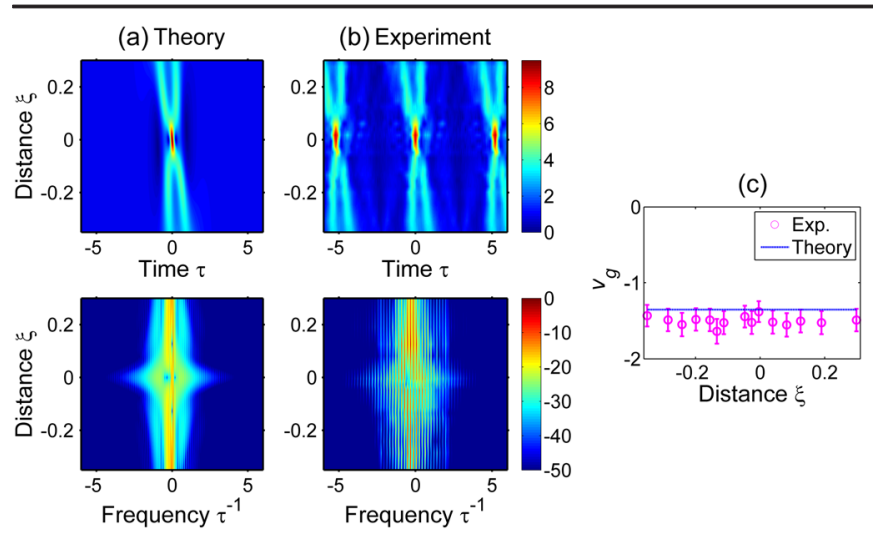

FIG. 4. Evolution of the breather molecule around node 2 in the temporal (top panels: amplitude profiles $|\psi|$ ) and spectral (bottom panels: power spectrum in $\mathrm{dB}$ scale) domains. (a) Two-breather solution. (b) Experimental wave measurements. (c) Comparison of the mean group velocity of the breather molecule obtained from spectral measurements to theoretical $V_{g_{-} \text {mol }}$ (error bars indicate the impact of limited OSA resolution). Breather parameters are the same as in Fig. 3.

the evolution of the envelope and its corresponding power spectrum.

In our experiments, breathers propagate with a group velocity different from the one of the background wave (i.e., breathers acquire a negative temporal shift to the line $\tau=0$, as shown in Fig. 3), but this cannot be retrieved directly from temporal measurements due to synchronization change between each fiber segment. We then determined the group velocity of breathers from spectral measurements to reconstruct exactly their space-time evolution reported in Figs. 3(c) and 4(b). By combining the dispersion relation of the optical fiber and the measured asymmetry power spectrum $S(\xi)=|\tilde{\psi}(\xi, \omega)|^{2}$, the group velocity of the breather molecule can be approximatively evaluated as $\left.\left.V_{g_{-} \text {mol }}(\xi) \approx \beta_{2}\right] \bar{\omega}(\xi)-\omega_{0}\right]$, where $\bar{\omega}(\xi)=$ $\left[\int S(\xi) \omega d \omega\right] /\left[\int S(\xi) d \omega\right]$ is the weighted average angular frequency, and $\omega_{0}$ is the carrier angular frequency of the background wave. Figure 4(c) shows the comparison between this experimental estimation and the theoretical prediction of Eq. (2). We retrieve a constant value of $V_{g_{\_} \text {mol }}$ as a function of propagation distance and close to the theoretical prediction. Here the discrepancies mainly come from the limited resolution of our optical spectrum analyzer equal to $2.5 \mathrm{GHz}$ when compared to the low group velocity to estimate, but also from the slight impact of the steady background wave.

Finally, to reveal the synthesis of breather molecule, additional measurements of the two individual breathers and their interactions with different temporal separations $\left(\mu_{1}-\mu_{2}=\pi, \pi / 2, \pi / 4\right.$ and $\left.\pi / 8\right)$ are presented in the Supplemental Material [42]. We also point out that numerical simulations based on the NLSE (1), taking into account the experimental conditions of periodic wave shaping and the fiber loss as well, were also performed. Corresponding results are not shown here, since it would be almost indistinguishable from experimental ones.

Conclusion.-In summary, we reported the theoretical description and direct observation of the synthesis and propagation of breather molecules, which can be fully characterized by the two-breather solution of the NLSE. More complex NLS photonic molecules can be formed by multiple breathers $(>3)$. The two-breather molecule exhibits much more complicated behavior, when compared to the bound state of two solitons. In the latter case soliton oscillations are simply periodic, while in the case of the breather molecule the exact periodic behavior is observed only when the commensurate condition is satisfied. It is also worth mentioning that this particular case of commensurate frequencies which we found is a potential description of the local modulation instability problem and related Fermi-Pasta-Ulam-Tsingou recurrence [43-45]. According to the universal applicability of NLSE, our results pave the way for novel directions of studies in the rich landscape of complex localized structures in wave physics, such as hydrodynamics, plasmas physics, and Bose-Einstein condensations. To conclude, our approach may stimulate future extensions of the notion of breather molecules towards dissipative systems, such as optical resonators and lasers in which multisoliton molecules are now extensively studied.

B. K. acknowledges financial support of the French "Investissements d'Avenir" program (PIA2/ ISITE-BFC, Contract ANR-15-IDEX-03, Project "Breathing Light"). V.Z. was supported by state assignment "Dynamics of the complex materials." A.G. is grateful for the support of the RFBR Research Project No. 16-31-60086 mol_a_dk. The theoretical part of this work was supported by the Russian Science Foundation (Grant No. 17-71-10128 to A. G.).

*bertrand.kibler@u-bourgogne.fr

These authors contributed equally to this work.

[1] A. C. Scott, The Nonlinear Universe: Chaos, Emergence, Life (Springer, Berlin, 2007).

[2] M. Remoissenet, Waves Called Solitons: Concepts and Experiments (Springer, Berlin, 1999).

[3] T. Dauxois and M. Peyrard, Physics of Solitons (Cambridge University Press, Cambridge, England, 2006).

[4] N. J. Zabusky and M. D. Kruskal, Phys. Rev. Lett. 15, 240 (1965).

[5] N. Akhmediev and A. Ankiewicz, Dissipative Solitons: From Optics to Biology and Medicine (Springer, Berlin, 2008).

[6] N. Akhmediev and A. Ankiewicz, Dissipative Solitons, Lecture Notes in Physics (Springer, Berlin, 2005).

[7] P. Grelu and N. Akhmediev, Nat. Photonics 6, 84 (2012).

[8] P. Grelu, Nonlinear Optical Cavity Dynamics: From Microresonators to Fiber Lasers (Wiley, Weinheim, 2016). 
[9] G. Herink, F. Kurtz, B. Jalali, D. R. Solli, and C. Ropers, Science 356, 50 (2017).

[10] K. Krupa, K. Nithyanandan, U. Andral, P. Tchofo-Dinda, and P. Grelu, Phys. Rev. Lett. 118, 243901 (2017).

[11] P. Ryczkowski, M. Närhi, C. Billet, J.-M. Merolla, G. Genty, and J. M. Dudley, Nat. Photonics 12, 221 (2018).

[12] X. Liu, X. Yao, and Y. Cui, Phys. Rev. Lett. 121, 023905 (2018).

[13] J. Peng, M. Sorokina, S. Sugavanam, N. Tarasov, D. V. Churkin, S. K. Turitsyn, and H. Zeng, Commun. Phys. 1, 20 (2018).

[14] G. I. Stegeman and M. Segev, Science 286, 1518 (1999).

[15] M. Stratmann, T. Pagel, and F. Mitschke, Phys. Rev. Lett. 95, 143902 (2005).

[16] U. Khawaja and H. Stoof, New J. Phys. 13, 085003 (2011).

[17] J. Javaloyes, M. Marconi, and M. Giudici, Phys. Rev. Lett. 119, 033904 (2017).

[18] Q.-Y. Liang, A. V. Venkatramani, S. H. Cantu, T. L. Nicholson, M. J. Gullans, A. V. Gorshkov, J. D. Thompson, C. Chin, M. D. Lukin, and V. Vuletić, Science 359, 783 (2018).

[19] N. Akhmediev and A. Ankiewicz, Chaos 10, 600 (2000).

[20] V. E. Zahkarov and A. B. Shabat, Zh. Eksp. Teor. Fiz. 61, 118 (1971) [Sov. Phys. JETP 34, 62 (1972)].

[21] D. H. Peregrine, J. Aust. Math. Soc. Series B, Appl. Math. 25, 16 (1983).

[22] Y. S. Kivshar and G. P. Agrawal, Optical Solitons: From Fibers to Photonic Crystals (Academic Press, London, 2003).

[23] M. J. Ablowitz, D. J. Kaup, A. C. Newell, and H. Segur, Phys. Rev. Lett. 30, 1262 (1973).

[24] D. Pelinovsky and R. Grimshaw, Phys. Lett. A 229, 165 (1997).

[25] A. V. Slyunyaev, J. Exp. Theor. Phys. 92, 529 (2001).

[26] N. Akhmediev and A. Ankiewicz, Solitons: Nonlinear Pulses and Beams (Chapman \& Hall, London, 1997).
[27] E. A. Kuznetsov, Dokl. Akad. Nauk SSSR 236, 575 (1977) [Sov. Phys. Dokl. 22, 507 (1977)].

[28] Y. C. Ma, Stud. Appl. Math. 60, 43 (1979).

[29] M. Tajiri and Y. Watanabe, Phys. Rev. E 57, 3510 (1998).

[30] V. E. Zakharov and A. A. Gelash, Phys. Rev. Lett. 111, 054101 (2013).

[31] A. Gelash and V. Zakharov, Nonlinearity 27, R1 (2014).

[32] N. Akhmediev, J. M. Soto-Crespo, and A. Ankiewicz, Phys. Lett. A 373, 2137 (2009).

[33] A. Chabchoub, M. Onorato, and N. Akhmediev, Lect. Notes Phys. 926, 55 (2016).

[34] B. Kibler, J. Fatome, C. Finot, and G. Millot, Lect. Notes Phys. 926, 89 (2016).

[35] J. M. Dudley, F. Dias, M. Erkintalo, and G. Genty, Nat. Photonics 8, 755 (2014).

[36] N. Bélanger and P. A. Bélanger, Opt. Commun. 124, 301 (1996).

[37] B. Malomed, Phys. Rev. A 44, 6954 (1991).

[38] N. Akhmediev, A. Ankiewicz, and J. Soto-Crespo, Phys. Rev. Lett. 79, 4047 (1997).

[39] B. Kibler, A. Chabchoub, A. Gelash, N. Akhmediev, and V. E. Zakharov, Phys. Rev. X 5, 041026 (2015).

[40] V. E. Zakharov and L. A. Ostrovsky, Physica (Amsterdam) 238D, 540 (2009).

[41] A. A. Gelash, Phys. Rev. E 97, 022208 (2018).

[42] See Supplemental Material at http://link.aps.org/ supplemental/10.1103/PhysRevLett.122.084101 for the derivation of characteristic frequencies of nonlinear Schrödinger two-breather molecules and the investigation of the temporal matching between two copropagating breathers.

[43] M. Conforti, S. Li, G. Biondini, and S. Trillo, Opt. Lett. 43, 5291 (2018).

[44] E. Kuznetsov, JETP Lett. 105, 125 (2017).

[45] A. Mussot, C. Naveau, M. Conforti, A. Kudlinski, F. Copie, P. Szriftgiser, and S. Trillo, Nat. Photonics 12, 303 (2018). 